\title{
A novel physical layer scheme based on superposition codes
}

\author{
Pietro Savazzi* (1) and Anna Vizziello
}

\begin{abstract}
The recently proposed superposition codes (SCS) have been mathematically proved to be decoded at any rate below the capacity, for additive white Gaussian noise (AWGN) channels. The main objective of this paper is to study the feasibility of a novel SC approach as an alternative to the traditional way of designing modern physical (PHY) layer schemes. Indeed, currently, PHY solutions are based on the decomposition into two separate problems of modulation shaping and coding over finite alphabets. Since superposition codes are defined over real numbers, modulation and coding can be jointly realized. Moreover, a fast decoding method is developed and tested by comparing the obtained results with both the uncoded system performance and two approximate message passing (AMP) algorithms. Finally, possible perspective to fifth generation (5G) applications exploiting SC solutions are outlined in the paper, and some interesting relations with sparse signal recovery are analyzed for further future research lines.
\end{abstract}

Keywords: Superposition code, Physical layer

\section{Introduction}

Some recent works have proven that sparse superposition codes (SCs), defined over real numbers, can be decodable at any rate less than the Shannon capacity with an exponentially small probability of error $[1,2]$. In more details, a maximum likelihood decoding allows reliable communications for all rates up to the Shannon capacity [1], while a fast decoding algorithm and its soft decoding version are proposed in [2] and [3] respectively.

According to the traditional information theory, the maximum transmission rate over an additive white Gaussian noise (AWGN) channel is [4]:

$$
C=\frac{1}{2} \log _{2}\left(1+\frac{P}{\sigma^{2}}\right)
$$

where $P$ is the codeword power and $\sigma^{2}=\frac{N_{0}}{2}$ is the Gaussian noise variance.

After the fundamental work of Shannon, the communication model for standard physical (PHY) layer design is usually split into two separate blocks: the channel coder and the modulation shaping [5]. Further, the best channel codes that may be found in the literature are based

*Correspondence: pietro.savazzi@unipv.it

Department of Electrical, Computer and Biomedical Engineering, University of Pavia, via Ferrata 5, Pavia, Italy on elements defined over binary or $q$-ary Galois finite fields [6].

Although the original Shannon proof of the noisy channel coding theorem is based on considering large and random codes defined over the real numbers set [7], the numerical instability and the power constraints of real number error-correcting codes have limited their use in practical communication schemes. On the other hand, they have been mainly studied for tackling fault tolerance problems in digital computers operation [8].

Moreover, some important and interesting research lines have been outlined $[9,10]$ by approaching the channel coding problem with codeword elements defined over the real or complex field. In the last decade, complex-field coding has been mainly used for counteracting multipath fading in wireless channels [11].

In [12], a low complexity coding scheme for RFID transmissions has been proposed, considering a simple implementation of a similar superposition coding idea. Despite its simple implementation, the codec scheme can provide a significant coding gain.

Sparse superposition codes can be thought as a joint modulation-coding scheme, since the main idea is to add redundancy directly to a spread spectrum signal, by assigning different orthogonal spreading sequences to the same link. 
This aspect could be exploited for the fifth generation (5G) technologies [13], especially from the point of view related to the interference rejection potential embedded in the SC scheme, which may be used for both fullduplex communications and non-orthogonal multi-access protocols that are considered in $5 \mathrm{G}$ systems.

The main contributions of this paper are as follows:

- The investigation-from an application-oriented point of view-of the novel SC concept, in order to test its feasibility and possible performance, gains with respect to the state-of-the-art PHY design. While [1-3] analyze SC from a theoretical perspective, we develop a simulation study case, which is still missing, useful to understand the benefits of SC application.

- A fast and simple decoding algorithm, which is less parameter-dependent compared to [2] and with a less computational burden with respect to the technique presented in [14].

- An overview about some interesting perspectives for future $5 \mathrm{G}$ research and development exploiting SC concept.

The proposed decoding scheme represents the most relevant novelty of this work, and it may be interpreted as an orthogonal matching pursuit (OMP) sparse reconstruction algorithm, while its limited number of iterations may be thought as a simple stopping rule [15].

This algorithm is similar to those shown in [2], although the two approaches differ for the following point: at each iteration, we look for the maximum values of the vector $Z^{(i)}$, see Eq. 7 and step 4 of the proposed algorithm in Section 3, while in [2], the elements of the same vector are compared to a specific threshold parameter $\tau$, see sections I.B and II of the cited reference.

Moreover, in Section II of [2], a combined statistics of the residuals $Y^{(i)}$, see Eq. 11, and a set of pacing parameters are used to theoretically compute the performance limit of the decoder. On the contrary, we have considered performance results obtained by simulations of the complete concatenated scheme.

Differently, in [1], the authors consider the performance limits of the optimal maximum likelihood detector, while in [3] a soft-decision decoder is taken under consideration with Bayes optimal statistics at each iteration. Finally, the works [14, 16-18] take into account approximate message passing decoding algorithms.

The paper is organized as follows: Section 2 is devoted to defining the superposition code and describing the system model. Section 3 details the proposed decoding scheme. Section 4 shows some interesting simulation results. Section 5 outlines relevant future 5G developments exploiting SCs and their relations with other approaches. Finally, some concluding remarks wrap up and close the paper in Section 6.

\section{Sparse superposition codes}

\subsection{The coding algorithm}

We here briefly outline the main concepts of SCs. The main insight of $[1,2]$ is to use superposition codes as a forward error correction (FEC) technique. In particular, we limit our analysis to binary transmission over an AWGN channel, although an extension to a non-binarysigned finite alphabet is possible to develop more spectral efficient schemes.

Specifically, let $u=\left(u_{1}, u_{2}, \ldots, u_{k}\right)$ be a string of $k$ bits to be coded. The main idea is to map $u$ to a vector $c$ of length $N$, whose elements are real numbers. Thus, let us define the dictionary matrix $X=\left[X_{1}, X_{2}, \ldots, X_{N}\right] \in$ $\mathbb{R}^{n \times N}$, whose entries are $X_{i, j} \sim \mathcal{N}(0,1)$, where $\mathcal{N}(0,1)$ represents a normal process with unit variance and zero mean. The codeword length equals $n$, so the code rate is $R=k / n$.

In order to obtain the codeword, a sparse vector $\beta \in \mathbb{R}^{N}$ is built as shown in Fig. 1. The matrix $X$ is partitioned into $L=N / M$ sections, being $L$ and $M$ positive integers. The codewords are computed as $c=X \beta^{T}$, with $\beta$ belonging to the set:

$$
\begin{aligned}
\mathcal{B}=\left(\beta: \beta=\sqrt{\frac{P}{L}} \text { in only one of the } M\right. \text { elements } \\
\text { of each section })
\end{aligned}
$$

Although [2] shows that rates closer to capacity at higher SNRs need an exponential power allocation profile, we use a constant power allocation. Indeed, in this way, we achieve even error probability along the codeword and a decoder with less parameters to be set than the scheme in [2], as explained in Section 3.

The detailed coding process is done as in the following:

- An input bit string $u$ of length $k=L \log _{2}(M)$ is split into $L$ substrings of size $\log _{2}(M)$

- Each substring of $u$ is to be intended as the position index of the non-zero element of $\beta$ in each of the $L$ subsections

- The rate of the superposition code, i.e., the number of input bits per channel use, can be then computed as:

$$
R=\frac{L \log _{2} M}{n}
$$

\subsection{System model}

The received sequence at the output of the AWGN channel is:

$$
Y=c+\eta=X \beta^{T}+\eta
$$

where $\eta$ is the noise vector whose elements belong to $\mathcal{N}\left(0, \sigma^{2}\right)$. 


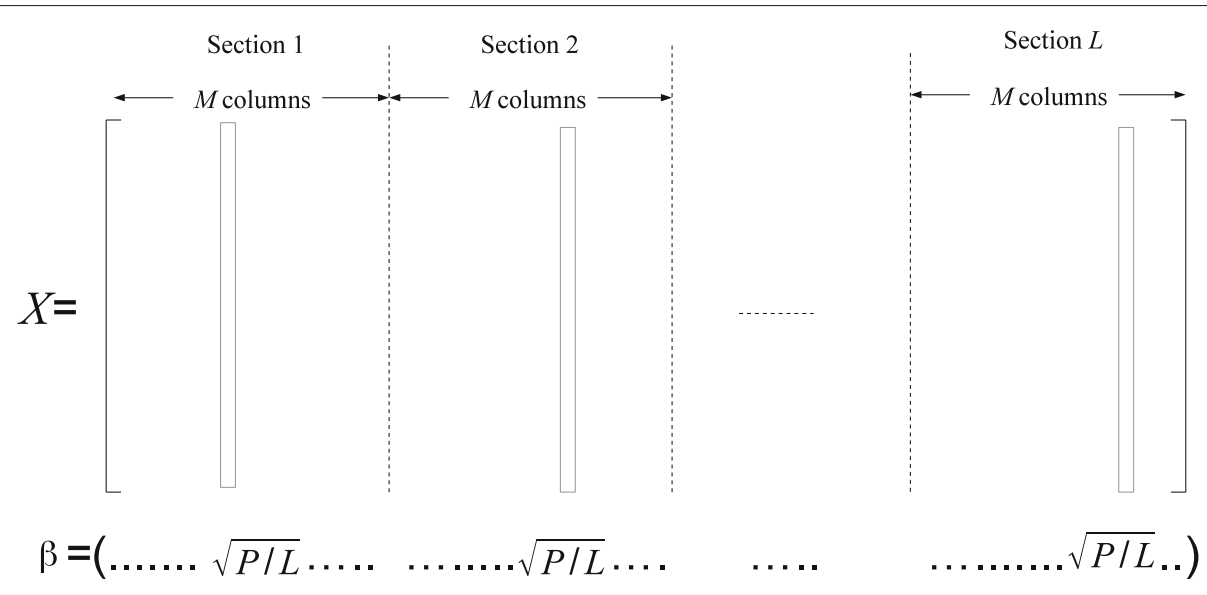

Fig. 1 Construction of the sparse vector $\beta$ as reported in [1]

According to the previous definitions, the codeword power has to be $P$, since:

$$
\|\beta\|^{2}=P \Rightarrow \frac{E\left(\left\|X \beta^{T}\right\|^{2}\right)}{n}=P
$$

Being $T$ the bit duration, the energy per bit to noise power spectral density ratio can be defined as:

$$
\frac{E_{b}}{N_{0}}=\frac{R P T}{\sigma^{2}}=\frac{R P}{N_{0} / 2}
$$

\section{The decoding algorithm}

We here propose a novel fast and simple decoding scheme for superposition codes. Similarly to the algorithm in [2], we propose an iterative decoding algorithm with a maximum number of iterations equal to $L$. However, the proposed solution is able to decode anyway the received codeword, without considering the possibility to mark erasures at the channel output as in [2]. In this way, the decoder always tries to output the more reliable decoded codeword.

This reasoning is consistent with the case study of FEC implementation, for which a decoder output may be always needed.

1. Initialize: $X^{(1)}=X, Y^{(1)}=Y, \hat{\beta}=0_{N}$,

$$
J^{(1)}=\left[J_{1}, J_{2}, \ldots, J_{L}\right] \text {, }
$$

where $J_{t}=[(t-1) M+1,(t-1) M+2, \ldots, t M]$ are the indexes of each of the $L$ sections of length $M$

2. For $i=1$ to $L$

3. Compute the vector $Z^{(i)}$ as in the following:

$$
Z^{(i)}=\frac{X^{(i) T} Y^{(i)}}{\left\|Y^{(i)}\right\|}
$$

4. Find the index $p$ of the maximum positive value of the vector $Z^{(i)}$. Since we only look for the maximum value of the vector $Z^{(i)}$, the normalization by $\left\|Y^{(i)}\right\|$ may be neglected and Eq. (7) may replaced by the simpler $Z^{(i)}=X^{(i) T} Y^{(i)}$

5. Identify the section $J_{t_{p}}^{(i)}$ containing the position $p$ and update the partially decoded word:

$$
\hat{\beta}(p)=\sqrt{(P / L)}
$$

6. if $i<L$, update:

$$
\begin{aligned}
& X^{(i+1)}=\left\{X_{l \in J_{i} \backslash \bigvee_{p}^{(i)}}^{(i)}\right\} \\
& J_{i+1}=J_{i} \backslash J_{t_{p}}^{(i)} \\
& Y^{(i+1)}=Y^{(i)}-X^{(i)} \beta^{T}
\end{aligned}
$$

7. Return to step (2).

After step (7), the vector $\beta$ contains the sparse representation of the decoded word.

Since at each iteration step we choose one of the $L$ groups corresponding to $\log _{2} M$ decoded bits, according to the position of the maximum of $Z^{(i)}$, after $L$ iterations the algorithm outputs the complete decoded word.

Once the decoding procedure of the joint demodulation-decoding scheme is completed, a further concatenated code can be used to correct any remaining residual errors, such as a Reed Solomon (RS) code [6] defined over $G F(M)[1,2]$, in order to improve the error correction performance. The use of inner and outer codes is a common practice in modern communication standards, since they allow different levels of error protection depending on transmitted data requirements or channel conditions.

Matching the Galois field size with the section dimension $M$ allows viewing each integer number belonging to the interval $[1, M]$ as a symbol in $G F(M)$.

This way of matching the Galois field size with a particular dimension of the inner concatenated scheme is similar in nature with the approach proposed in $[19,20]$. 
Since $L \leq M$ [1], a shortened Reed Solomon [6] $(L, L-2 t)$ code can be obtained from an $\left(n_{R S}, k_{R S}\right)$ one, so that the total code rate of the concatenated scheme is:

$$
R=\frac{k(L-2 t)}{n L}
$$

where $n_{R S}-k_{R S}=2 t$ is even.

\section{Simulation results}

Simulation results have been obtained by using the set of parameters listed in Table 1 . We have chosen a standardde-facto 255,239 Reed Solomon shortened code, as the outer scheme. This choice influenced the other parameter settings as explained in the following.

- The number of bits $\log _{2} M$ in each of the $L$ subsections has been set equal to 8 , corresponding to $M=256$, in order to match the symbol dimension of the outer Reed Solomon code, as explained at the end of the previous section.

- Since $k=L \log _{2} M$, we have chosen the number of subsections $L=128$ in order to have a moderate codeword length equal to 2048 for a rate of $R=0.5$.

- This value of $L$ also satisfies the restricted isometry property (RIP) for random sensing, see Eq. (13) of [21], for which $L \leq \frac{n}{C \log M}$. For instance, that corresponds to $L<369$ for $n=2048$, and the constant $C=1$.

- The constant power allocation is determined by $P=\frac{E_{b}}{L T}$, where $E_{b}$ is the average signal energy per bit and $T$ is the bit duration.

As shown in Figs. 2 and 3, the performance of the proposed scheme looks promising, especially for the SC code rate equal to $\frac{1}{3}$, shown in Fig. 2. The SC gain for the code rate $R=\frac{1}{2}$ could be improved by optimizing the code parameters, following for instance the procedure described in [2]. In particular, the code length could be chosen larger in order to improve the decoder performance, since the parameter $L$ could be increased up to 256 , keeping satisfied the RIP condition for $R=0.5$.

Table 1 Simulation parameter setting

\begin{tabular}{ll}
\hline Simulation parameters & \\
\hline $\mathrm{L}$ & 128 \\
$\mathrm{M}$ & 256 \\
Code rate SC code & $1 / 3,1 / 2$ \\
$\left(n_{R S}, k_{R S}\right)$ & $(255,239)$ \\
$\mathrm{RS} t$ & 8 \\
Code rate of the concatenated scheme & $0.29167,0.4375$ \\
Message length $k$ & 1024 \\
Codeword length $n$ & 3072,2048 \\
\hline
\end{tabular}

In Fig. 4, the frame error rate (FER) of the proposed scheme is compared to those obtained by the approximate message-passing decoding algorithms presented in $[14,18]$. The rate of all the schemes is 0.5 , while the code parameters for the two AMP decoders are the same shown in Fig. 2 of [16], according to the code A case.

Although the proposed decoder performance is a little worse with respect the one obtained by the algorithm presented in [14] by Rush et al., the presented results are anyway interesting since they needed an extremely lower computational burdening. This may be appreciated by considering the computational complexity analysis in Table 2, where we compare the number of algebraic operations required by the proposed scheme and the AMP shown in [14] and analyzed in Table II of [16].

For the computational comparison analysis, we have only considered the AMP algorithm of [14] since it is more similar to the presented scheme, considering both implementation and performance. Anyway, it is possible to note that the actual complexity of the proposed algorithm is comparable to a simple correlation receiver for code division multiple access (CDMA) signals; nevertheless, the obtained coding gain is comparable to those attainable by more complex techniques.

\section{Overview on 5G applications}

The main drivers of the current research for $5 \mathrm{G}$ are Internet of Things (IoT), Gigabit wireless connectivity, and Tactile Internet among others [22], which call for several communication requirements. The underlying design principles, i.e., synchronism and orthogonality, of the PHY layer of the current Long-Term Evolution Advanced (LTE-A) radio access network constitute the major drawbacks for the conceived service architecture. On this purpose, as stated in [22], new waveforms are envisioned as possible solutions. In this section, we outline possible applications of this work on SCs to different aspects of wireless communications, with particular emphasis to 5G applications.

\subsection{Compressed sensing}

Sporadic traffic-generating devices (e.g., machine-type communication (MTC) devices in 5G IoT) awake now and then, thus they should transmit their data immediately and only coarsely synchronized, conversely to the fine synchronization procedure of LTE-A PHY layer random access [22]. On this purpose, sparse signal processing and new waveforms can be combined to efficiently deal with the sporadic traffic and control signaling in the uplink random access channel (RACH) [22]. The sparse representation of superposition codes, and sparse signal processing methodology in general, are effective for this application. Indeed, the detection and demodulation of MTC traffic in the PHY layer RACH benefits from the "bursty" nature 


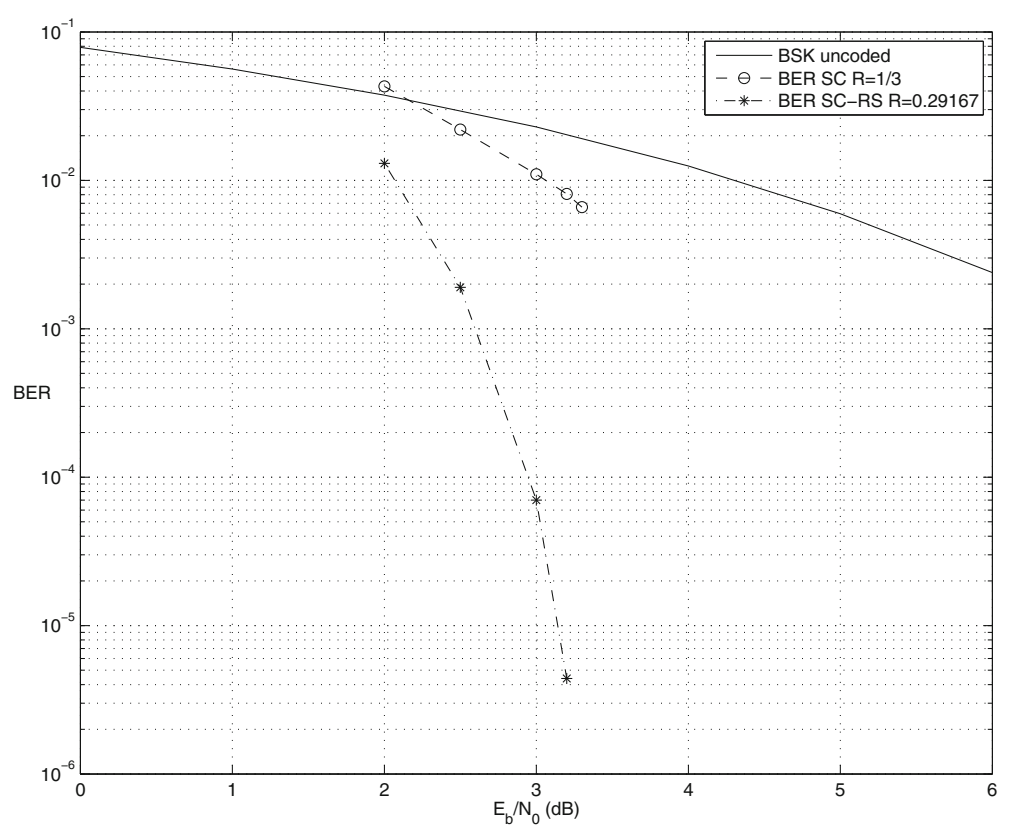

Fig. 2 BER versus $\frac{E_{b}}{N_{0}}$ for the uncoded, SC, and SC-RS architectures. The code rate is 0.29167

of signals ("sparse" in mathematical sense) and they can be described by a small set of parameters within a larger set of observables [22-24]. A possible solution is to make sparse signal processing usable for 5G RACH, exploiting joint sparsity of messages, mobile channels, and user activity [22].

\subsection{Extension to more spectrally efficient schemes}

Given the increasing amount of traffic data for 5G services, spectrally efficient solutions become mandatory. Although we will limit our analysis on SC to a preliminary binary case, it would be possible to increase the SC spectral efficiency by extending the presented results to the

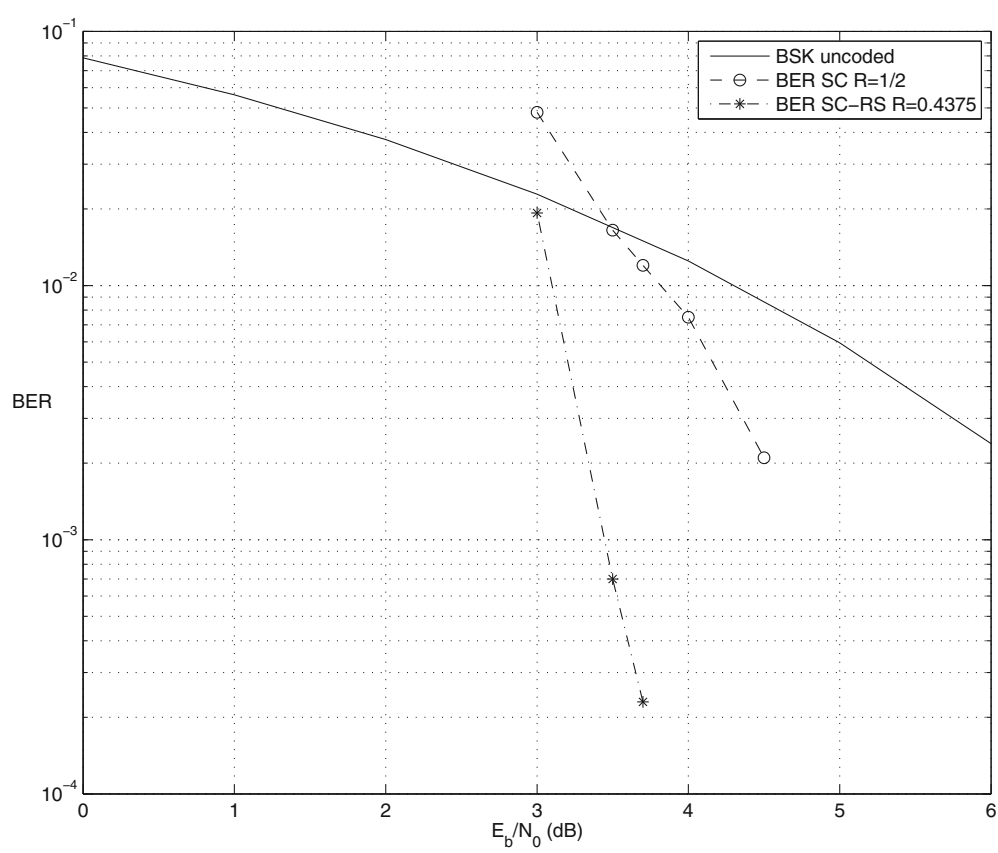

Fig. 3 BER versus $\frac{E_{b}}{N_{0}}$ for the uncoded, SC, and SC-RS architectures. The code rate is 0.4375 


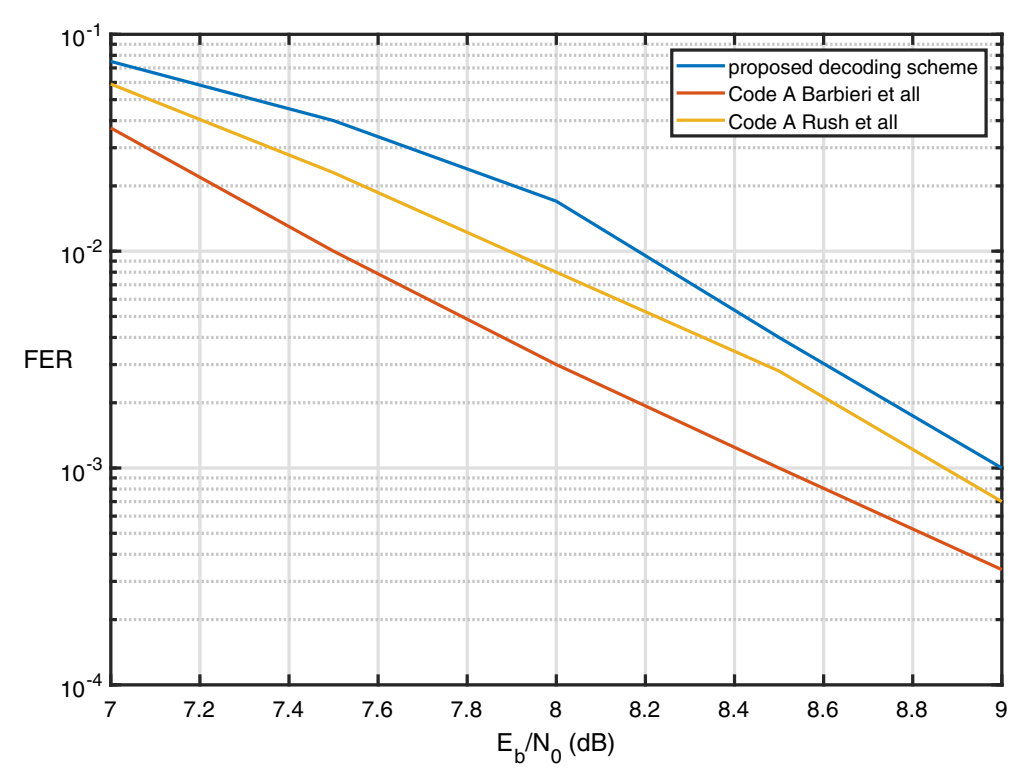

Fig. 4 FER versus $\frac{E_{b}}{N_{0}}$ comparison between the proposed scheme and two examples of SC decoded by the AMP algorithm. The code rate is 0.5 for all the three schemes

non-binary case. For instance, in [25] and [26], a signed superposition coding scheme is proposed, and in [27], an algorithm is developed to determine both the locations and amplitudes of the non-zero elements in the sparse vector.

\subsection{Multiple access scheme}

Superposition codes have been firstly introduced in [28] for multi-user channels, in order to compute the capacity region of the Gaussian broadcast channel [1]. In this sense, a similar scheme applies where the multiple user information is both superimposed and coded simultaneously for downlink multiple access channels.

Furthermore, this novel PHY technique could be suitable for non-orthogonal multiple access schemes [29, 30], since SC schemes can be interpreted as signal despreading with error correction capabilities, which could make non-orthogonal multiple access receivers more robust to user interferences. This would be useful also for $5 \mathrm{G}$

Table 2 Computational complexity comparison

\begin{tabular}{lll}
\hline $\begin{array}{l}\text { Number of operations per } \\
\text { each iteration }\end{array}$ & Proposed decoder & AMP, Rush et al. [14] \\
\hline Sum & $2 M L(n-1)+n$ & $2 M L(n+1)-n-L$ \\
Multiplication & $n(2 M L+1)$ & $n(2 M L+1)+3 M L$ \\
Division & - & $2 M L+n+1$ \\
Exponential & - & $M L$ \\
\hline
\end{tabular}

solutions, in particular for full-duplex links where the self-interference level is very severe.

\subsection{Synchronization}

Synchronism is the underlying design principles of the PHY layer of today's LTE-A radio access network [22]; thus, novel synchronization solutions for the future 5G service architecture will be really useful. On this purpose, thinking about SC codes as a novel method for both modulation and coding, at least for the inner code, an interesting perspective is to develop joint algorithm for decoding and channel parameter synchronization to be applied to schemes like the ones in [31,32].

In more details, the code structure, i.e., the sensing matrix $X$ which is known at the receiver, may be used for both frame synchronization and fine clock recovery, instead of using pilot symbols. In this way, the spectral efficiency may be improved by decreasing the number of required pilots.

\section{Conclusions}

In this work, we have highlighted the feasibility of real number superposition coding. This field, closely related to compressed sensing algorithms, seems to be very promising because the simple decoding algorithm could be efficiently applied to very high data-rate signals, by using a mixed digital-analog system. This can relax the analog-todigital conversion requirements since the algorithm acts on real numbers. On this purpose, further deep investigations will be essential. 
Ongoing research works are devoted to extend the proposed scheme to non-binary signaling techniques in order to improve the final spectral efficiency. Furthermore, a real implementation in a software-defined-radio (SDR) platform will enable a more precise characterization of the true system performance, by carefully considering the synchronization related issues.

An important objective will be to look for performance as close as possible to channel capacity with dictionaries of moderately small sizes, allowing small signal spaces. From a communication theory point of view, starting from [3], new probabilistic algorithms capable of achieving performances closer to channel capacity may be sought. We believe that concatenation with a robust outer code, including Reed Solomon and $q$-ary LDPC, shall be necessary in order to obtain good performance.

\section{Authors' contributions}

Both the authors have conceived the proposed algorithm and developed the MATLAB code used for producing the presented results. The final manuscript has been written and revised by the two authors. Both authors read and approved the final manuscript.

\section{Competing interests}

The authors declare that they have no competing interests.

\section{Publisher's Note}

Springer Nature remains neutral with regard to jurisdictional claims in published maps and institutional affiliations.

Received: 27 January 2017 Accepted: 10 August 2017

Published online: 29 August 2017

\section{References}

1. A Joseph, AR Barron, Least squares superposition codes of moderate dictionary size are reliable at rates up to capacity. IEEE Trans. Inf. Theory. 58(5), 2541-2557 (2012)

2. A Joseph, AR Barron, Fast sparse superposition codes have near exponential error probability for $\mathrm{R}<$ C. IEEE Trans. Inf. Theory. 60(2), 919-942 (2014)

3. AR Barron, S Cho, in Proc. IEEE Int. Symp. on Information Theory (ISIT). High-rate sparse superposition codes with Iteratively Optimal Estimates (IEEE, Cambridge, 2012)

4. CE Shannon, A mathematical theory of communication. Bell Syst. Tech. J. 27(5), 379-423623656 (1948)

5. GD Forney Jr., G Ungerboeck, Modulation and coding for linear Gaussian channels. IEEE Trans. Inf. Theory. 44(6), 2384-2415 (1998)

6. S Lin, DJ Costello, Error Control Coding, Second Edition. (Prentice-Hall, Inc, Upper Saddle River, 2004)

7. CE Shannon, Communication in the presence of noise. Proc. IRE. $\mathbf{3 7}$ (1), 10-21 (1949)

8. Z Chen, in Proceedings of the Conference on High Performance Computing Networking, Storage and Analysis. SC'09. Optimal real number codes for fault tolerant matrix operations (ACM, New York, 2009), pp. 29-12910

9. TG Marshall Jr., Coding of real-number sequences for error correction: a digital signal processing problem. IEEE J Sel. Areas Commun. 2(2), 381-392 (1984)

10. TH Liew, LL Yang, L Hanzo, Systematic redundant residue number system codes: analytical upper bound and iterative decoding performance over AWGN and Rayleigh channels. IEEE Trans. Commun. 54(6), 1006-1016 (2006)

11. Z Wang, GB Giannakis, Complex-field coding for OFDM over fading wireless channels. IEEE Trans. Inf. Theory. 49(3), 707-720 (2003)

12. E Goldoni, P Savazzi, A Vizziello, A novel channel coding scheme for rfid generation-2 systems. Electronics. 6(1), 1-9 (2017). http://www.mdpi.com/ 2079-9292/6/1/4, doi:10.3390/electronics6010004

13. IF Akyildiz, S Nie, S-C Lin, M Chandrasekaran, 5 g roadmap: 10 key enabling technologies. Comput. Netw. 106, 17-48 (2016)
14. C Rush, A Greig, R Venkataramanan, Capacity-achieving sparse superposition codes via approximate message passing decoding. IEEE Trans. Inf. Theory. 63(3), 1476-1500 (2017)

15. $\Pi$ Cai, L Wang, Orthogonal matching pursuit for sparse signal recovery with noise. IEEE Trans. Inf. Theory. 57(7), 4680-4688 (2011)

16. C Condo, WJ Gross, in 2015 IEEE Workshop on Signal Processing Systems (SiPS). Sparse superposition codes: a practical approach (IEEE, Hangzhou, 2015), pp. 1-6

17. C Condo, WJ Gross, Implementation of sparse superposition codes. IEEE Trans. Signal Proc. 65(9), 2421-2427 (2017)

18. J Barbier, F Krzakala, in 2014 IEEE International Symposium on Information Theory. Replica analysis and approximate message passing decoder for superposition codes (IEEE, Honolulu, 2014), pp. 1494-1498

19. A Marinoni, P Savazzi, P Gamba, Efficient detection and decoding of q-ary Idpc coded signals over partial response channels. EURASIP J. Wirel. Commun. Netw. 2013(1), 18 (2013)

20. A Marinoni, P Savazzi, in Proc. IEEE International Conference on Communications (ICC). Ecient receivers for q-ary LDPC coded signals over partial response channels (IEEE, Cape Town, 2010)

21. EJ Candes, MB Wakin, An introduction to compressive sampling. IEEE Signal Proc. Mag. 25(2), 21-30 (2008)

22. G Wunder, P Jung, M Kasparick, T Wild, F Schaich, Y Chen, ST Brink, I Gaspar, N Michailow, A Festag, L Mendes, N Cassiau, D Ktenas, M Dryjanski, S Pietrzyk, B Eged, P Vago, F Wiedmann, 5GNOW: non-orthogonal, asynchronous waveforms for future mobile applications. IEEE Commun. Mag. 52(2), 97-105 (2014)

23. HF Schepker, C Bockelmann, A Dekorsy, in 2013 IEEE 77th Vehicular Technology Conference (VTC Spring). Coping with cdma asynchronicity in compressive sensing multi-user detection (IEEE, Dresden, 2013), pp. 1-5

24. H Zhu, GB Giannakis, Exploiting sparse user activity in multiuser detection IEEE Trans. Commun. 59(2), 454-465 (2011)

25. AC Gilbert, JA Tropp, in Proc. IEEE International Symposium on Information Theory (ISIT). Applications of sparse approximation in communications (IEEE, Adelaide, 2005)

26. JA Tropp, Just relax: convex programming methods for identifying sparse signals in noise. IEEE Trans. Inf. Theory. 52(3), 1030-1051 (2006)

27. DL Donoho, A Maleki, A Montanari, Message-passing algorithms for compressed sensing. Proc. Natl. Acad. Sci. 106(45), 18914-18919 (2009)

28. TM Cover, Broadcast channels. IEEE Trans. Inf. Theory. 18(1), 2-14 (1972)

29. P Wang, J Xiao, L Ping, Comparison of orthogonal and non-orthogonal approaches to future wireless cellular systems. IEEE Veh. Technol. Mag. 1(3), 4-11 (2006)

30. P Wang, J Xiao, L Ping, Correction to "Comparison of Orthogonal and Non-Orthogonal Approaches to Future Wireless Cellular Systems". EEE Veh. Technol. Mag. 1(4), 42 (2006)

31. P Savazzi, P Gamba, L Favalli, An iterative algorithm for joint symbol timing recovery and equalization of short bursts. J. Commun. 3(4), 34-40 (2008)

32. P Savazzi, P Gamba, Iterative symbol timing recovery for short burst transmission schemes. IEEE Trans. Commun. 56(10), 1729-1736 (2008)

\section{Submit your manuscript to a SpringerOpen ${ }^{\circ}$ journal and benefit from:}

- Convenient online submission

- Rigorous peer review

- Open access: articles freely available online

- High visibility within the field

- Retaining the copyright to your article

Submit your next manuscript at $\boldsymbol{\nabla}$ springeropen.com 